\title{
Dual-adjuvant effect of pH-sensitive liposomes loaded with STING and TLR9 agonists regress tumor development by enhancing Th1 immune response
}

\author{
Banu Bayyurt Kocabas ${ }^{\mathrm{a}, 1}$, Kubra Almacioglu ${ }^{\mathrm{a}}$, Esin Alpdundar Bulut ${ }^{\mathrm{b}}$, Gozde Gucluler ${ }^{\mathrm{a}}$, \\ Gizem Tincer ${ }^{\mathrm{a}}$, Defne Bayik ${ }^{\mathrm{c}}$, Mayda Gursel ${ }^{\mathrm{b}}$, Ihsan Gursel ${ }^{\mathrm{a}, *}$ \\ ${ }^{a}$ Thorlab. Therapeutic ODN Research Lab, Department of Molecular Biology and Genetics, Bilkent University, Bilkent, 06800 Ankara, Turkey \\ ${ }^{\mathrm{b}}$ Department of Biological Sciences, Middle East Technical University, 06800 Ankara, Turkey \\ ${ }^{\mathrm{c}}$ Lerner Research Institute, Cleveland Clinic, Cleveland, OH 44195, USA
}

\section{A R T I C L E I N F O}

\section{Keywords:}

CpG ODN

cGAMP

pH-sensitive liposome

Immune-adjuvant synergy

Cancer immunotherapy

\begin{abstract}
A B S T R A C T
Nucleic acid-based pattern recognition receptor agonists are effective adjuvants and immunotherapeutic agents. Rather than single applications, ligand combinations could synergistically potentiate immune responses by elevating cytokine and chemokine production via triggering multiple signaling pathways. However, short halflives of such labile ligands due to nuclease attack and limited cellular uptake due to their structure significantly hamper their in vivo performances. More importantly, simultaneous delivery and activity presentation of protein antigen and nucleic acid ligands critically limit the clinical development of these constructs. In this work, we approached this problem by co-encapsulating a model antigen ovalbumin along with TLR9 and STING ligands within liposomes, a well-established drug delivery system that enables payload stability and enhanced cellular activity upon internalization. Moreover, by loading dual ligands we postulated to achieve heightened Th-1 immune response that would yield pronounced protective vaccine efficacy. We show that, pH-sensitive liposomes co-encapsulating CpG ODN and cGAMP induced synergistic innate immune response by elevating type I and type II interferon levels. Most importantly, this vaccine formulation led to $\sim 70 \%$ regression of established melanoma tumor. $\mathrm{pH}$-sensitive liposomal vaccine administration elevated IgG2c/IgG1 antibody ratio, indicative of augmented OVA-specific Th1-biased immunity. Importantly, while the frequency of tumor-specific IFN- $\gamma$ producing $\mathrm{CD}^{+}$T-cells was significantly increased, the M2-type anti-inflammatory macrophage levels were decreased in the tumor bed. In conclusion, our strategy induces reversal of immunosuppressive tumor microenvironment, while enhancing effective anti-tumor immune-response. We propose that this could be coupled with standard therapies during combating tumor eradication.
\end{abstract}

\section{Introduction}

Immunotherapy is a promising approach that aims to strengthen the immune system against tumors that evade immune surveillance and induce tolerance. Recent efforts have focused on ways to stimulate immunity more effectively to provide immune-mediated elimination of tumors using delivery technologies which decrease the side effects and improve the potency of immunotherapy [1].

Among immunostimulatory molecules, nucleic acid-based adjuvants are considered as potent and safe agents that initiate strong immune activation [2,3]. One of the most extensively studied nucleic acid is single stranded oligodeoxynucleotides (ODNs) expressing unmethylated CpG motifs (CpG ODNs) [4,5]. The most suitable ODN type for clinical use is K-type CpG ODN (also known as CpG B). Their recognition by TLR9 localized at the late endosomes activates NF- $\mathrm{KB}$ signaling and promotes pro-inflammatory cytokine secretion [6]. Their potential to maintain an immunological memory and to provide post-immunization has been demonstrated in prophylactic vaccines against anthrax and

\footnotetext{
Abbreviations: APCs, antigen presenting cell; BM-DC, Bone marrow-derived dendritic cell; BM-DM, Bone marrow-derived macrophage; CDN, cyclic dinucleotide;

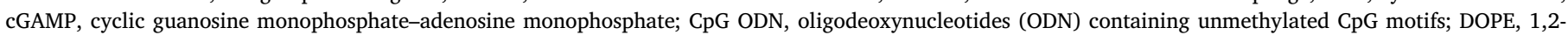

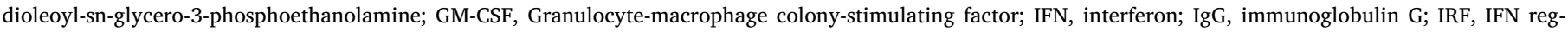

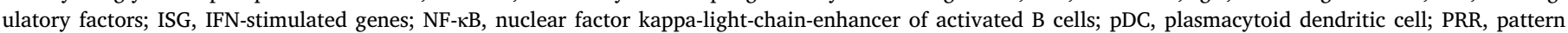
recognition receptor; SSCL, sterically-stabilized cationic liposomes; STING, stimulator of IFN genes; TBK1, TANK Binding Kinase 1; TLR, Toll-like receptor

* Corresponding author.

E-mail address: ihsangursel@bilkent.edu.tr (I. Gursel).

${ }^{1}$ Current affiliation: Laboratory of Host-Pathogen Dynamics, National Heart, Lung, and Blood Institute, NIH, Bethesda, MD 20892, USA.
} 
hepatitis B [7]. However, K-type ODNs are inefficient in therapeutic vaccines against cancer due to impotent stimulation of pDCs to secrete type I IFNs, which drives the generation of anti-tumoral activities [8]. To augment the adjuvant potential of K-type ODN, new strategies are being developed by combining or conjugating them with different immunostimulatory ligands [9], cationic peptides [10], or nanoparticles [11].

Previously, we demonstrated that the combination of CpG-ODN with STING agonist, which are cyclic dinucleotides (CDNs), enhanced its immunostimulatory properties [12]. STING is a endoplasmic reticulum-bound cytosolic protein, of which stimulation with CDNs such as cGAMP induces the expression of type I IFNs through TBK1 and IRF3 activation, and secretion of pro-inflammatory cytokines via NF-kB activation [13-15]. We have shown that simultaneous administration of CpG-ODN and CDNs triggers synergistic immune response by increasing cytokine production, elevating co-stimulatory molecule expression and antigen-specific adaptive immune responses [12]. This immune response initiated by activation of both MyD88 and TRIF dependent pathways. Besides, according to proposed pathway of synergism, TRAF6 recruitment to both pathways elevates interferons and proinflammatory cytokines. Confirming our observation, another study reported that cGAMP and CpG ODN combination has an anti-tumoral activity with increased type II IFN production by NK cells [16]. This elevation was IRF3/7, STING and MYD88 dependent. However, this synergy can be elicited at high adjuvant concentrations due to the low cellular uptake and rapid clearance of nucleic acids from circulation.

Considering these results, synergy might be dependent on amounts of adaptor molecules and transcription factors which can be increased by co-delivery of ligands into a single cell to maintain their crosstalk. To enhance their cellular uptake, stability in circulation and innate immune responses, based on our previous experiences on delivery of single and dual-TLR agonists [17-19], we co-encapsulated cGAMP and K3-type CpG ODN into modified sterically-stabilized cationic liposome (SSCL) and provided co-delivery to their target receptors. Due to their differential subcellular localization of TLR9 and STING, we aimed to use a pH-sensitive liposome. This provides both endosomal and intracellular delivery by inducing pore formation in endosome at acidic environment [20]. Our results revealed that this approach increased the immunostimulatory potential of CpG-ODN and cGAMP at doses that do not induce any immune responses when they are given as such in solution. Liposomal constructs elevated the production of both type I and type II IFNs and Th1-biased cytokine secretion from APCs. Furthermore, when employed as a therapeutic vaccine, liposomes significantly regressed B16 melanoma tumor growth by activating antigen-specific humoral and cellular immune responses, as well as reducing M2-type macrophage frequency in the tumor microenvironment.

\section{Materials and methods}

\subsection{Reagents}

All lipids were purchased from Avanti Polar Lipids (Alabaster, AL, USA) except cholesterol (Chol) (Sigma Aldrich, St. Louis, MO, USA). TLR9 ligand, CpG-ODN ATCGACTCTCGAGCGTTCTC was kindly provided by Dr. Ken Ishii (Tokyo Univ, Japan). CpG motif is underlined. Cy5-labeled CpG-ODN was synthesized in CBER/FDA core Facility and provided by Dr. Dennis M. Klinman. 2'3' cGAMP was purchased from Invivogen (Toulouse, France). FAM-labeled 2'3' cGAMPs was obtained from Biologs (Bremen, Germany). All cell culture media components were either from Gibco (NY, USA) or Lonza (Walkersville, MD, USA). Cytokine ELISA reagents: recombinant cytokines, monoclonal unlabeled and biotinylated antibodies against mIL-6, mIL-10 and mIL-12 and were purchased from Biolegend (San Diego, CA, USA), while streptavidin-alkaline phosphatase and $\mathrm{mIFN}-\gamma$ were purchased from Mabtech (Cincinnati, OH, USA). $P$-nitrophenyl phosphate disodium salt substrate (pNPP) was purchased from Thermo Scientific (USA).
Immunoglobulin ELISA reagents; goat anti-mouse total IgG, IgG1, IgG2c, monoclonal antibodies conjugated with alkaline phosphatase (AP) were from Southern Biotech (Birmingham, AL, USA). FACS antibodies conjugated to fluorescent chromophores were obtained from Biolegend. B16-Blue IFN- $\alpha / \beta$ cells that allow the detection of bioactive murine type I IFNs were obtained from Invivogen (Toulouse, France) and used according to the manufacturer's protocol.

\subsection{Liposome preparation}

DC-CHOL, DOPE, PEG-PE, PC and cholesterol were used to prepare SSCL (DC-CHOL:DOPE:PEG-PE) at different lipid ratios (high, medium, low; 3:7:0.06, 4:6:0.06, 5:5:0.06) and neutral liposome (CHOL:PC; 1:1) by dehydration-rehydration method as described earlier [17,18]. Briefly, lipids were mixed and film was formed using rotary evaporator (ILMVAC, Ilmenau, Germany). Vesicles were generated by hydrating film with $1 \times$ phosphate buffer solution (PBS; $\mathrm{pH}$ : 7.4). They were sonicated five times for $30 \mathrm{~s}$ on and $30 \mathrm{~s}$ off at $4{ }^{\circ} \mathrm{C}$ using a Vibra Cell Sonicator (Sonics and Materials, Danbury, CT, USA) to generate small unilamellar vesicles (SUV). Ligand loading within lipid vesicles were performed as described earlier [19]. Briefly, ligands (0.5 mg of ODN or $0.2 \mathrm{mg}$ of CDN or their $1: 3(\mathrm{w} / \mathrm{w})$ ratio) and preformed SUVs $(10 \mu \mathrm{mole})$ were mixed and snap frozen in liquid nitrogen, and freeze-dried overnight (VirTis benchtop K, Bieleveld, Germany). Dried mixture was rehydrated (1/10 initial volume) using nuclease-free $\mathrm{ddH}_{2} \mathrm{O}$ and vortexed for $15 \mathrm{~s}$ every $5 \mathrm{~min}$ for $30 \mathrm{~min}$ at room temperature. Equal volume of PBS was added to the mixture to adjust the tonicity of the resulting liposomes. Liposomes were washed twice with $1 \times \mathrm{PBS}(\mathrm{pH}: 7.4)$ to remove the unloaded molecules and centrifuged at $16,000 \times g$ for $1 \mathrm{~h}$. Liposome formulations were stored at $4{ }^{\circ} \mathrm{C}$ until use.

The encapsulation efficiency of liposomes was analyzed using liposomes encapsulating FITC-labeled CpG ODN and/or FAM-labeled cGAMP. Fluorescent non-encapsulated ligands were detected using spectrofluorometer (excitation at: $495 \mathrm{~nm}$; emission at: $515 \mathrm{~nm}$; BioTek, Model: Synergy HT). Encapsulation efficiency (EE) was calculated using the equation below:

$\% \mathrm{EE}=\left(\mathrm{C}_{\mathrm{i}}-\mathrm{C}_{0}\right) /\left(\mathrm{C}_{\mathrm{i}}\right) \times 100$

$\mathrm{C}_{\mathrm{i}}$ : initial amount; $\mathrm{C}_{0}$ : non-encapsulated amount.

\subsection{Characterization of $\mathrm{pH}$ sensitivity of liposome}

Calcein entrapment was performed by rehydrating $10 \mu$ mole lipid film with $1 \mathrm{ml} 80 \mathrm{mM}$ calcein in $1 \times$ PBS (Sigma Aldrich, St. Louis, MO, USA) during liposome preparation. As previously explained, liposome was prepared and centrifuged at $16,000 \times g$ for $1 \mathrm{~h}$ to remove excess amount of calcein. pH-dependent release was investigated incubating them in $1 \times$ PBS at different pHs: $4-7$ in a shaker $\left(100 \mathrm{rpm}, 37^{\circ} \mathrm{C}\right)$ for $1 \mathrm{~h}$. Leakage of calcein led de-quenching of dye and was detected using spectrofluorometer (excitation at: $495 \mathrm{~nm}$; emission at: $515 \mathrm{~nm}$; BioTek, Model: Synergy HT). Percentage was calculated using the equation below:

$\%=\left[\left(\mathrm{C}_{\mathrm{pH}}-\mathrm{C}_{0}\right) /\left(\mathrm{C}_{\mathrm{T}}-\mathrm{C}_{0}\right)\right] \times 100$

$\mathrm{C}_{0}=$ concentration of calcein in the buffer (pH: 7.4); $\mathrm{C}_{\mathrm{pH}}=$ concentration of calcein at different $\mathrm{pHs} ; \mathrm{C}_{\mathrm{T}}=$ total calcein after Triton X-100 was added.

\subsection{Generation of bone marrow-derived dendritic cells and macrophages}

Bone marrow-derived dendritic cells (BM-DCs) were generated from bone marrow isolated from the femur and tibia of C57BL/ 6 mice incubating with GM-CSF (25 ng/ml) and IL-4 (10 ng/ml) for 6-8 days. Immature BM-DCs were $80 \% \mathrm{CD}_{11} \mathrm{~b}^{+} / \mathrm{CD} 11 \mathrm{c}^{+}$double positive as previously demonstrated [17]. Bone marrow-derived macrophages (BM-DMs) were generated from bone marrow isolated from the femur 
and tibia of C57BL/6 mice incubated with 30\% L929-conditioned medium (L929-CM) for 6 days. Generated BM-DMs were F4/80 and CD11b double positive.

\subsection{In vitro stimulation}

Splenocytes $\left(2 \times 10^{6} \mathrm{cell} / \mathrm{ml}\right.$ in $\left.250 \mu \mathrm{l}\right)$ or bone marrow-generated cells $\left(0.5 \times 10^{6}\right.$ cell $/ \mathrm{ml}$ in $\left.250 \mu \mathrm{l}\right)$ were stimulated with free or liposome loaded CpG-ODN (0.1-1 $\mu \mathrm{M})$ and/or cGAMP $(0.1-9 \mu \mathrm{M})$ for $24-36 \mathrm{~h}$ at $37{ }^{\circ} \mathrm{C}$ in a $5 \% \mathrm{CO}_{2}$ incubator. After stimulation, the supernatants were collected and used for cytokine ELISA and detection of type I IFNs by B16-Blue ${ }^{\mathrm{TM}}$ IFN- $\alpha / \beta$ reporter cells. For uptake and binding experiments, splenocytes were incubated with free or liposomeencapsulated Cy5-labeled CpG ODN and/or FAM-labeled cGAMP for $1 \mathrm{~h}$ at $37^{\circ} \mathrm{C}$. Uptake and binding percentages of cells were analyzed using BD Accuri C6 flow cytometer.

\subsection{Anti-tumoral activity of $C p G-O D N$ and cGAMP loaded liposome against melanoma}

Animals were kept in the animal holding facility of the Department of Molecular Biology and Genetics at Bilkent University under controlled ambient conditions $\left(22 \pm 2{ }^{\circ} \mathrm{C}\right)$ regulated with $12 \mathrm{~h}$ light and $12 \mathrm{~h}$ dark cycles. They were provided with unlimited access of food and water. The animal ethical committee of Bilkent University has approved all experimental procedures (Approval \#: BILAEC NO:2014/30).

Female C57BL/6 mice (6-8 week old) were inoculated subcutaneously with B16-OVA $\left(1 \times 10^{6} /\right.$ mice in $\left.100 \mu \mathrm{l} 1 \mathrm{xPBS}\right)$ at the right flank of mice on day 0 . Tumor volume (length $\mathrm{x}$ width $\mathrm{x}$ height) was determined measuring dimensions of tumor with electronic caliper. When tumor volume was around $50 \mathrm{~mm}^{3}$, they were treated with free or co-encapsulated CpG-ODN (5 $\mu \mathrm{g} /$ dose), cGAMP (1.7 $\mu \mathrm{g} / \mathrm{dose})$ and OVA ( $5 \mu \mathrm{g} /$ dose) administered intraperitoneally (i.p.) on specified days. Third and fourth injections contain double amounts of each adjuvant. At the end of the experiment, blood was collected from tail vein of mice and antigen-specific IgG subtypes (Total IgG, IgG1 and IgG2c) were analyzed by ELISA from collected sera (1/2000 diluted) of each sample. Collected tumors and spleens were weighted. Excised tumor tissues were digested in Liberase DL (Roche Applied Science, Mannheim, Germany) and stained with fluorochrome-labeled anti-mouse Abs to determine percentages of infiltrating cells. Splenocytes were incubated with OVA or SIINFEKL to detect antigen specific IFN- $\gamma$ production by cytokine ELISA or antigen specific $\mathrm{CD}^{+} \mathrm{T}$ cell levels by ELISPOT.

\subsection{Flow cytometry analysis}

Single cells isolated from tumor tissues were filtered through $40 \mu \mathrm{m}$ cell strainer and red blood cells were lysed using ACK lysis buffer (Walkersville, MD, USA). Cells were stained with fluorochrome-labeled antibody cocktail including mouse redFluor 710-CD45, PB-TCR- $\beta$, FITC-CD4, APC-CD8, PE-B220, APC-NK1.1, FITC or PE-CD11b, PE-Gr1, and PerCP/Cy5.5-CD206 and analyzed using CytoFlex flow cytometer (Beckman Coulter, Brea, CA, USA). Zombie Aqua Fixable Viability Kit (Biolegend, San Diego, CA, USA) was used to determine live/dead cells. Gating strategy was shown in Fig. S1. Data were analyzed using FlowJo software (Tree Star, OR, USA).

\subsection{ELISA and ELISPOT assay}

Immulon $2 \mathrm{HB}$ or 1B microtiter plates (Thermo Scientific, San Jose, CA, USA) were coated with anti-cytokine or anti-IgG abs, respectively, and then blocked with PBS-BSA. Serially diluted standards and culture supernatants or serially diluted mouse sera were added to plates for overnight at $4{ }^{\circ} \mathrm{C}$. Cytokine was detected using biotinylated anti-cytokine Abs followed by phosphatase-streptavidin whereas bound IgG subclasses were detected using alkaline phosphatase streptavidin conjugated anti-IgG antibodies and developed by the addition of pNPP substrate. Anti-IFN- $\gamma$ Ab coated Immulon $2 \mathrm{HB}$ plates were blocked with PBS-BSA in ELISPOT assay as previously explained [17].

\subsection{Statistical analysis}

Statistical significant differences between groups were determined using one-way ANOVA following Tukey's post-hoc comparison or twoway ANOVA following Bonferroni's post-test, and Student's t-test analysis using GraphPad Prism software (version 5, San Diego, CA, USA). $P<0.05$ was considered as significant.

\section{Results}

\subsection{DOPE containing liposomes deliver the cargo in a pH dependent manner}

We prepared sterically-stabilized cationic liposomes (SSCL) using DOPE, which is a fusogenic lipid, for our drug release system. To assess the pH-sensitivity, we entrapped a fluorescent dye, calcein that has a self-quenching property at high concentrations. It can be detected when released from the delivery system via spectrofluorometer (ex: $495 \mathrm{~nm}$; em: $515 \mathrm{~nm}$ ). Control liposome that contains no DOPE constantly released calcein at similar percentages in $1 \times$ PBS at different pHs. However, we observed that SSCL formulations prepared with different DOPE ratios (low, medium and high) leaks calcein in a pH-dependent manner. Higher leakage was at lower pHs $(5.6 \pm 4.6 \%$ calcein leakage at $\mathrm{pH}: 7.0$ vs $53.2 \pm 10.2 \%$ calcein leakage at $\mathrm{pH}: 4.0 ; p<0.001$; Fig. 1A).

These $\mathrm{pH}$ sensitive liposomes have a spherical structure with around 400-700 nm size of which changes according to their cargo (Fig. S2; Table 1). In addition, encapsulation efficiency of CpG-ODN and cGAMP

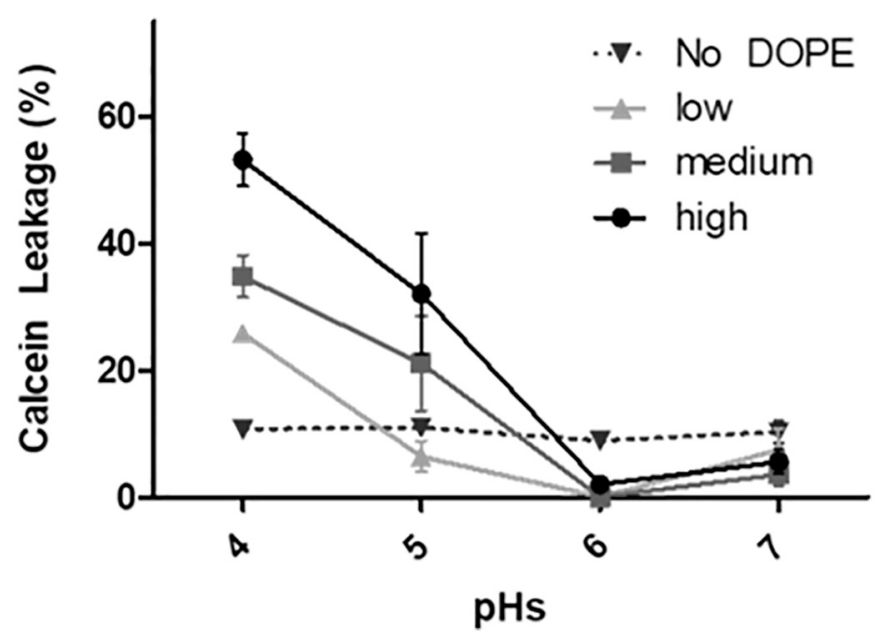

Fig. 1. Characterization of $\mathrm{pH}$ sensitive liposome. Liposomes (SSCL; DCCHOL:DOPE:PEG-PE at different lipid ratios: high, medium, low; 3:7:0.06, 4:6:0.06, 5:5:0.06 respectively, and no DOPE; CHOL:PC; 1:1) were prepared using different amounts of $\mathrm{pH}$-sensitive lipid, DOPE. Calcein, a fluorescent dye that has a self-quenching property at high concentrations was entrapped into liposomes. (A) $\mathrm{pH}$ dependent release mechanism of liposomes was investigated incubating them in 1xPBS at different pHs: 4-7 in a shaker $\left(100 \mathrm{rpm}, 37^{\circ} \mathrm{C}\right.$ ) for $1 \mathrm{~h}$. Dequenching of calcein was detected via spectrofluorometer (ex: $495 \mathrm{~nm}$, em: $515 \mathrm{~nm})$. The calcein leakage percentage was calculated as $=\left[\left(\mathrm{C}_{\mathrm{pH}}-\mathrm{C}_{0}\right) /\right.$ $\left.\left(\mathrm{C}_{100}-\mathrm{C}_{0}\right)\right] \times 100 . \mathrm{C}_{0}=$ concentration of calcein in the buffer before incubation. $\mathrm{C}_{\mathrm{pH}}=$ concentration of calcein at different pHs after incubation. $\mathrm{C}_{100}=$ total concentration of calcein after Triton X-100 treatment of samples. Samples were run in triplicates and experiment repeated twice. Error bars represent SEM. Statistical significance was determined by two-way ANOVA followed by Bonferroni (HSD) test. ${ }^{* *} p<0.001$; ns: non-significant. 
Table 1

Size and polydispersity index (PDI) of pH-sensitive liposomes loaded with CpG$\mathrm{ODN}$ and/or cGAMP (mean $\pm \mathrm{SD}$ ).

\begin{tabular}{lll}
\hline & Size $(\mathrm{nm})$ & PDI \\
\hline Empty SSCL & $401 \pm 14$ & $0.262 \pm 0.008$ \\
(CpG-ODN)SSCL & $754 \pm 45$ & $0.148 \pm 0.016$ \\
(cGAMP)SSCL & $636 \pm 43$ & $0.036 \pm 0.015$ \\
(CpG-ODN + cGAMP)SSCL & $674 \pm 74$ & $0.209 \pm 0.011$
\end{tabular}

in co-encapsulated form was $85.6 \pm 0.4 \%$ and $93.8 \pm 0.8 \%$, respectively.

For the rest of our study, we chose to use liposomes containing the highest DOPE ratio to load our adjuvants. According to our calcein release experiment and previous studies, we contemplated that liposomes will stay intact at neutral pHs while they will release their cargo at acidic environment (lysosome or late endosome) due to destabilization of its structure [20].

\subsection{Production of type I and type II IFNs from splenocytes stimulated with} $p H$-sensitive liposome co-encapsulating $C p G-O D N$ and $c G A M P$

To understand whether our new liposomal adjuvant co-encapsulating CpG-ODN and cGAMP can enhance innate immune responses, we tested them on splenocytes by incubating either with free or liposome-encapsulated CPG ODN and/or cGAMP at different concentrations for $36 \mathrm{~h}$. Supernatants were used to detect IFN- $\gamma$ and IFN- $\alpha$ /

A
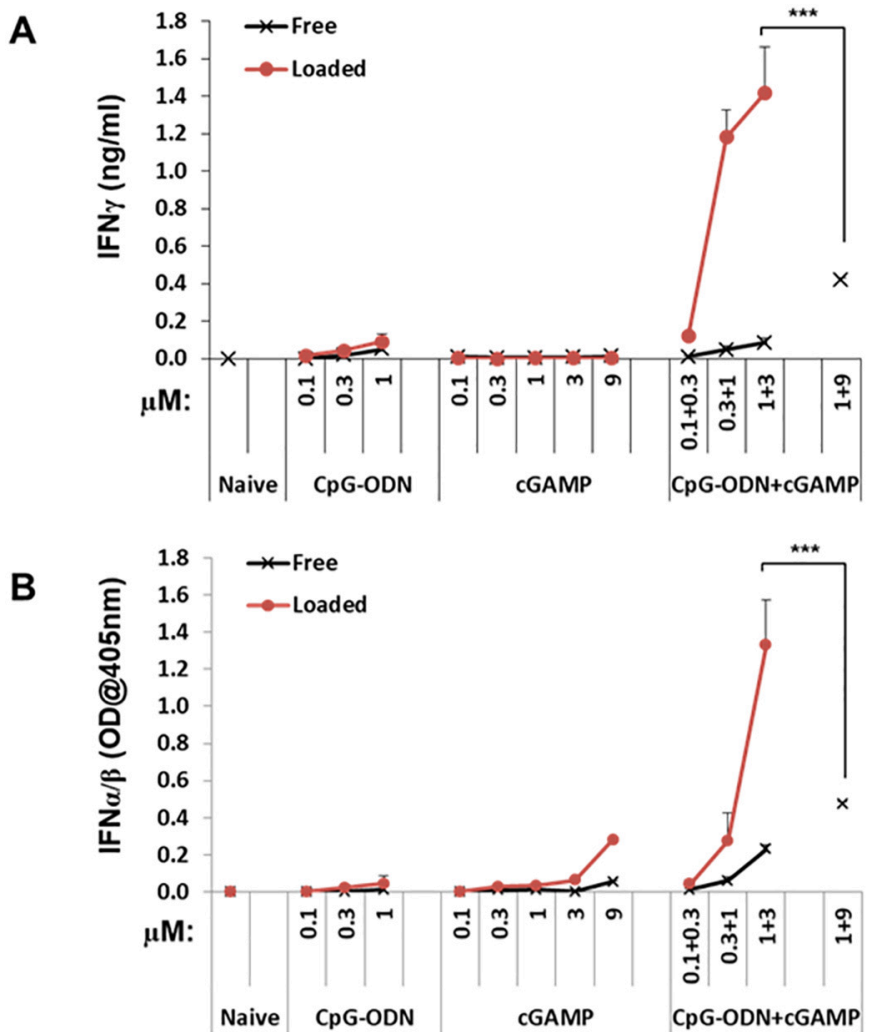

Fig. 2. Interferon secretion from splenocytes stimulated with free or liposomeloaded CpG-ODN and/or cGAMP. Splenocytes were stimulated with free or liposome-loaded CpG-ODN and/or cGAMP for $36 \mathrm{~h}$. Supernatants were collected and (A) IFN- $\gamma$ and (B) IFN- $\alpha / \beta$ levels were detected via ELISA and reporter cell line (B16-Blue ${ }^{\mathrm{TM}}$ IFN- $\alpha / \beta$ Cells), respectively. Samples were run in triplicates and experiments were repeated twice. Error bars represent SEM. Statistical significance was determined by one-way ANOVA followed by Tukey's (HSD) test, $* * * p<0.001$. (For interpretation of the references to colour in this figure legend, the reader is referred to the web version of this article.) $\beta$ levels by ELISA and B16-Blue ${ }^{\mathrm{TM}}$ IFN- $\alpha / \beta$ reporter cells (Fig. 2). As previously shown by Yildiz et al., only high doses of free CpG-ODN $(1 \mu \mathrm{M})$ plus cGAMP $(9 \mu \mathrm{M})$ combination significantly elevate IFN- $\gamma$ and IFN- $\alpha / \beta$ production compared to adjuvant groups with single ligands at the same concentrations $(p<0.001$, one-way ANOVA, Tukey's posthoc test) [12]. When CpG-ODN and cGAMP co-encapsulated into liposome, approximately 10-15 fold increase in the secretion of IFN- $\gamma$ ( $\mathrm{p}<0.001$, Fig. 2A) and 5-fold increase in the secretion of IFN- $\alpha / \beta$ compared to free combinations ( $\mathrm{p}<0.001$, Fig. $2 \mathrm{~B}$ ) at ratios that no significant interferon production was observed in free dual ligands.

When cell viability of splenocytes after treatment was measured using CCK- 8 assay, there was no difference between media treated or liposome treated cells (Fig. S3A).

\subsection{Cytokine secretion from APCs treated with liposome co-encapsulating $C p G-O D N$ and $c G A M P$}

Antigen presenting cells (APCs) characterize $\mathrm{T}$ cell differentiation and determine the polarization of immune system. After ascertaining that liposomes co-encapsulating CpG-ODN plus cGAMP combination on splenocytes synergistically activates Th1-biased immune response, we investigated their effects on dendritic cells and macrophages after $24 \mathrm{~h}$ of stimulation in culture. When generated BM-DCs with treatment of GM-CSF and IL-4 stimulated with free or liposome loaded agonists, formulation with co-encapsulated CpG-ODN $(1 \mu \mathrm{M})$ and cGAMP $(3 \mu \mathrm{M})$ significantly increased secretion of IL-6 (2-fold) and IL-12 (7-fold) compared to stimulation with free forms of dual ligands at the same concentrations ( $p<0.001$, Fig. 3).

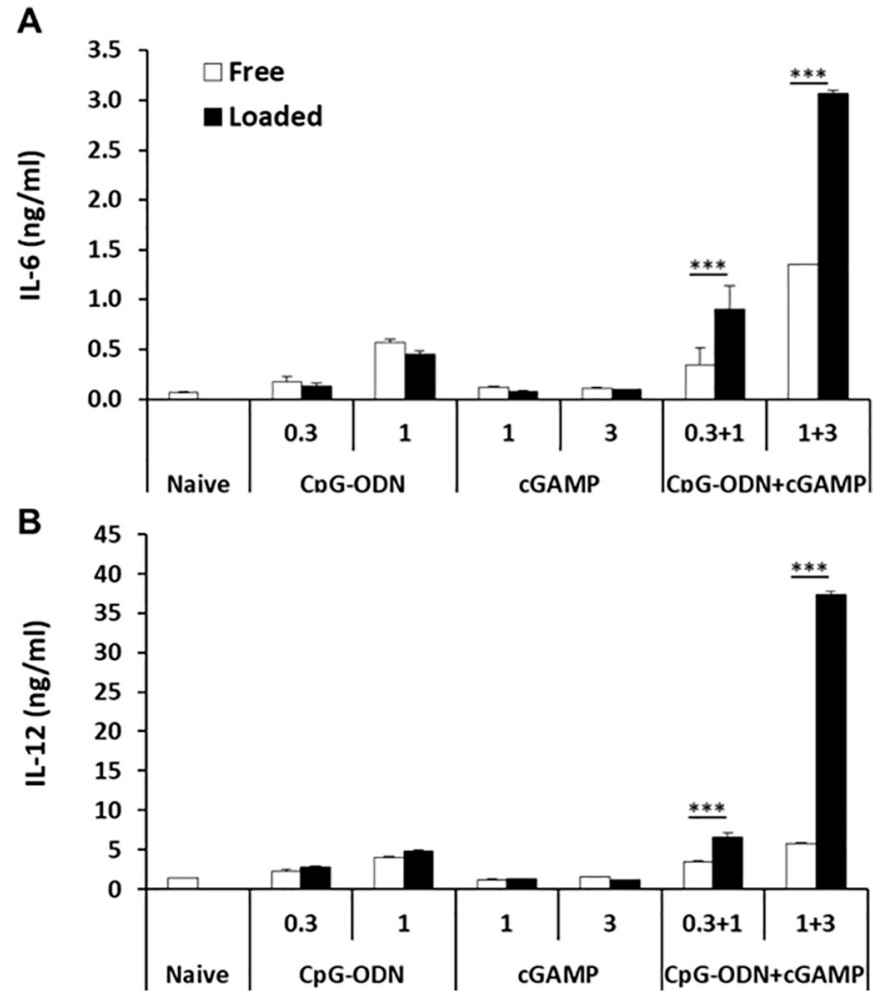

Fig. 3. Cytokine profile of bone marrow-derived dendritic cells (BM-DCs) activated with free CpG-ODN and/or cGAMP or co-encapsulated liposomes. BMDCs generated by incubating with GM-CSF and IL4 were stimulated with free or liposome encapsulated CpG-ODN and/or cGAMP for $24 \mathrm{~h}$. (A) IL-6 and (B) IL-12 amounts in supernatants were detected via ELISA. Samples were run in triplicates. Error bars represent SD. Experiment was repeated twice with similar results. Statistical significance was determined by one-way ANOVA followed by Tukey's (HSD) test, ${ }^{* * *} p<0.001$. 
Table 2

IL-12 and IL-10 produced by bone marrow-derived macrophages (BM-DM) stimulated with free or liposome-loaded CpG-ODN and/or cGAMP.

\begin{tabular}{|c|c|c|c|c|c|}
\hline Treatment & Dose $(\mu \mathrm{M})$ & & IL12 (ng/ml) & IL10 (ng/ml) & IL12/IL10 \\
\hline \multirow[t]{2}{*}{ CpG-ODN } & 1 & Free & $3.0 \pm 0.2$ & $23.7 \pm 0.5$ & 0.1 \\
\hline & & Liposome & $5.9 \pm 0.1$ & $9.3 \pm 3.6$ & 0.6 \\
\hline \multirow[t]{2}{*}{ cGAMP } & 3 & Free & $0.2 \pm 0.0$ & $0.0 \pm 0.0$ & - \\
\hline & & Liposome & $0.5 \pm 0.0$ & $0.0 \pm 0.0$ & - \\
\hline \multirow[t]{2}{*}{ CpG-ODN + cGAMP } & $1+3$ & Free & $26.1 \pm 3.0$ & $22.5 \pm 4.5$ & 1.2 \\
\hline & & Liposome & $153.1 \pm 1.9^{*}$ & $38.8 \pm 1.0$ & 4.0 \\
\hline
\end{tabular}

Cytokines secreted from BM-DMs following ligand treatments were presented in Table 2. It was observed that liposome co-encapsulating dual ligands secreted significantly higher amounts of IL-12 than IL-10. This data suggests that liposome co-encapsulating dual ligands generates higher frequency of M1 macrophages from M0-macrophages to that of M2 macrophage generation. Of note, IL-12/IL-10 ratio (IL-12 ${ }^{\text {hi }}$ / IL-10 ${ }^{\text {lo: }}$ M1; IL-12 ${ }^{\text {lo }} / \mathrm{IL}-10^{\text {hi }}$ : M2) was calculated to distinguish macrophage polarization.

Besides elevated Th1-biased cytokine secretion, we witnessed that treating liposomes co-encapsulating CpG ODN and cGAMP did not hamper macrophage viability (Fig. S3B).

\subsection{Cellular uptake of CpG ODN and cGAMP increases following $p H$ sensitive liposome loading}

To investigate the cellular uptake and binding of CpG ODN and cGAMP combination, we stimulated splenocytes with free or liposomeloaded Cy5-labeled CpG ODN $(1 \mu \mathrm{M})$ and/or FAM-labeled cGAMP $(3 \mu \mathrm{M})$. Fluorochrome positivity of cells was analyzed via flow cytometer (Fig. 4A and B). While uptake and binding of cGAMP or CpG ODN didn't change when simultaneously administered into cells, encapsulation of agonists, separately or together, into $\mathrm{pH}$-sensitive liposome increased their uptake. Relative MFI values of FAM and Cy5 in cells treated with co-encapsulated liposomal formulation were higher than cells treated with free combination ( 4 and 150 fold more, respectively, Fig. 4C).

\subsection{Anti-tumoral activity of liposome co-encapsulating $C p G$ ODN and cGAMP against established B16 melanoma}

Tumor surveillance would be activated and immune suppressive microenvironment in tumor would be cleared or repolarized into Th1biased immunity via immunotherapy. To assess whether our liposome co-encapsulated CpG ODN and cGAMP formulation has a potential as immunotherapeutic agent, a melanoma tumor model was established by inoculating ovalbumin (OVA)-expressing B16 cells (B16-OVA) to right flank of C57BL/6 mice subcutaneously (s.c.). When tumor volume reached approximately $50 \mathrm{~mm}^{3}$, mice were treated with free or liposome co-encapsulating CpG ODN and cGAMP together with OVA. Treatments were repeated at pre-determined time points (days pointed with arrows in Fig. 5).

While tumor growth kinetics was similar in untreated and free dual ligand treated groups, tumor size of mice treated with co-encapsulated liposome was significantly retarded $(p<0.05)$. At the end of the experiment, tumor weight was lower and spleen size was larger in liposome co-encapsulated group (Fig. S4A-B). Besides, no significant weight change was observed in mice throughout immunotherapy period (Fig. S4C).

3.6. Enhanced antigen-specific humoral and cellular adaptive immune response by liposome co-encapsulating $C p G$ ODN and cGAMP

To assess humoral responses, OVA-specific total IgG, IgG1 and IgG2c were measured via ELISA from sera of mice collected at day 18 in

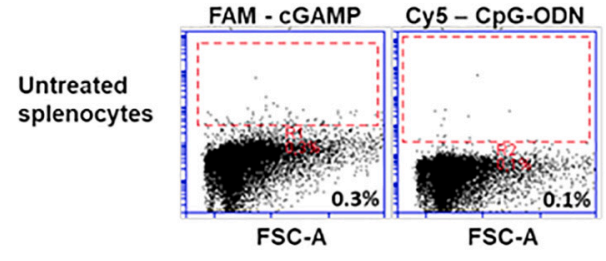

A



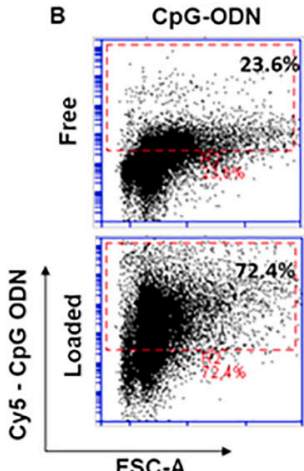

C

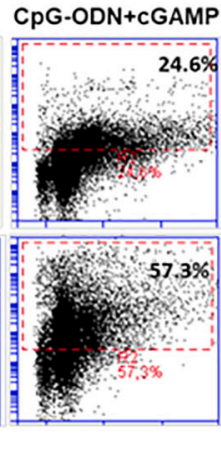

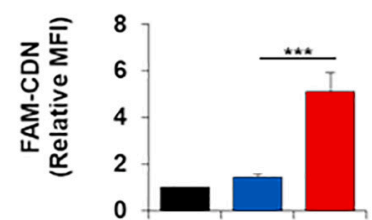

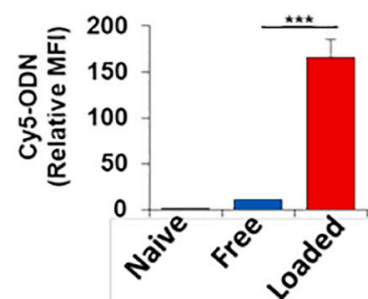

Fig. 4. Cellular uptake of free or co-encapsulated CpG ODN + cGAMP. Splenocytes were incubated with Cy5-labeled CpG ODN (1 $\mu$ M) and FAM-labeled cGAMP $(3 \mu \mathrm{M})$ for $1 \mathrm{~h}$ and uptake and binding level was detected via flow cytometer. Representative dot plots of (A) FAM-labeled cGAMP, (B) Cy5-labeled CpG ODN, and (C) relative MFI values comparing unencapsulated free ligands vs loaded ligands are presented. Samples were run in triplicates. Error bars represent SD. Experiments were repeated twice with similar results. Statistical significance was determined by one-way ANOVA followed by Tukey's (HSD) test, ${ }^{* * *} p<0.001$. 


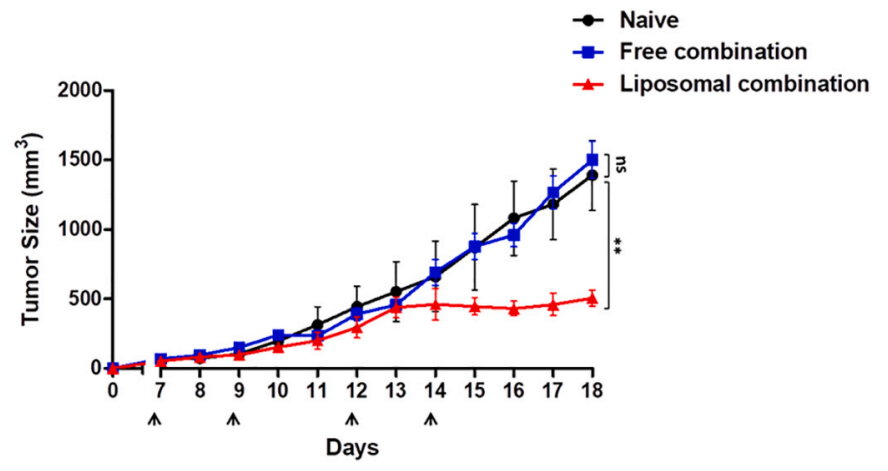

Fig. 5. PH-sensitive CPG ODN and cGAMP co-encapsulated SSCL in tumor immunotherapy. C57BL/6 mice ( $n=5$ /group) were inoculated subcutaneously on day 0 with B16-OVA-luc $\left(1 \times 10^{6} /\right.$ mice). When tumor volumes were $\sim 50 \mathrm{~mm}^{3}$, animals were administered intraperitoneally either saline (Black line), or free (Blue line) or co-encapsulated (Red line) CpG ODN (5 $\mu \mathrm{g} / \mathrm{animal})$ and cGAMP ( $1.7 \mu \mathrm{g} /$ animal) plus OVA ( $5 \mu \mathrm{g}$ /animal) into liposomes ( 0.1 $\mu$ mole) on days indicated with arrows. Error bars represent SEM. $p$-value was determined by two-way ANOVA followed by Bonferroni (HSD) test comparing treatment groups with untreated (naive) group. ns: non-significant, $* * p<0.01$. (For interpretation of the references to colour in this figure legend, the reader is referred to the web version of this article.)

tumor-bearing mice (Fig. 6A). As expected, there was no OVA-specific antibody production in naive animals. Besides, treatment with $\mathrm{CPG}$ ODN and cGAMP co-encapsulated within liposomes elevated OVAspecific total IgG and IgG2c production much higher than free combination treated mice. We detected significantly elevated IgG2c/IgG1 ratio after treatment with liposomal formulations, indicating the development of antigen specific Th1-biased immunity $(p<0.05$, Fig. 6B).

Antigen-specific IFN- $\gamma$ production was detected by ELISA after incubating splenocytes with model antigen OVA for $24 \mathrm{~h}$ in naive and ligand treated B16 melanoma inoculated mice. Consistent with tumor volumes, IFN- $\gamma$ levels were higher in splenocytes of mice treated with liposome-loaded CpG ODN and cGAMP ( $p<0.05$, Fig. 6C). In addition, splenocytes were incubated with SIINFEKL peptide and IFN- $\gamma$ producing $\mathrm{CD}^{+} \mathrm{T}$ cells were determined via ELISPOT. Likewise with OVA incubation, liposome co-encapsulating CpG ODN and cGAMP significantly elevated IFN- $\gamma^{+} \mathrm{CD}^{+}$T cells $(p<0.001$, Fig. 6D).

The success of cancer immune therapy is not solely dependent on the elicited tumor antigen specific immunity. The alteration of the immunosuppressive tumor microenvironment to a more inflammatory milieu is another critical step for ensuring eradication/regression of the tumor mass. To further investigate tumor infiltrating immune cell identities, we collected tumors and digested them with liberase enzyme. Immune cell percentages in tumors were evaluated using flow cytometer. While tumor-associated macrophages (TAMs), myeloid-derived suppressor cells (MDSCs), and granulocytes were in similar percentages in tumors of naive and treated mice (data not shown), liposomal dual agonist treatment significantly reduced frequency of tumor-resident M2-macrophages ( $p<0.05$, Fig. 7). Additionally, nos2 expression was similar in all treatments while arg expression in tumor tissue of mice treated with liposomal dual ligands was lower than the naive group (Fig. S5).

\section{Discussion}

Innate immune system initiates multiple signaling cascades during a pathologic insult. This activation is dependent on simultaneous engagement of different ligands to their corresponding receptors either existing on the cell surface or present within the endosomes or cytoplasmic space. In some instances, PRRs might cross-talk to either boost or suppress the overall resultant pathogen-specific innate immune response [21,22]. We previously demonstrated that the activation of MyD88-dependent and -independent pathways using a combination of STING and TLR9 agonists generates synergistic immune response [12]. Cytosolic DNA induces STING-mediated immune activation initiated by CDNs, whereas CpG ODN in endosome leads to TLR9 mediated immune response. STING agonist (3'3'-cGAMP) is prone to trigger a Th2-biased immune response and K-type CpG ODN has low effects on type I IFN secretion. The combination of ligands enhanced type I IFN, IL-6, IP-10 and TNF- $\alpha$, secretion and augmented the efficiency of agonists as antitumor immunotherapeutic agents. Consistent with our observations, this synergistic activity between STING and TLR9 pathways has also been reported by Temizoz et al. as an increase in type-II IFN production particularly by NK cells [16].

Although these findings point to potential benefits of combining multiple agonists; like CpG ODNs, free CDNs have restricted clinical use. Poor cytosolic uptake, insufficient migration to lymph nodes due to their low molecular weights and instability in circulation limit their immune stimulatory properties in vivo. For this reason, recent efforts are focused on designing delivery tools to enhance the stable internalization proportions of such labile molecules to their target cells/ tissues and facilitate their subcellular localization [23-27]. It has been well established that $\mathrm{pH}$-sensitive liposomes deliver their cargo into cytosol [28]. Miyabe et al. (2015) used this feature to efficiently deliver the STING agonist, $c$-di-GMP by loading into $\mathrm{pH}$-sensitive liposomes containing YSK05 lipid with high fusogenic property. Consequently with increased cytosolic uptake, this system increased IFN- $\beta$ production, activated NK cells, and provided anti-tumor effect on lung metastasis of B16-OVA cells [26,27].

Co-encapsulation is a strategy which ensures that both TLR9 and STING ligands and the antigen are uptaken by the same cell. This method spares both antigen and adjuvant. Several studies showed that co-encapsulated formulation has a superior immune response than separately loaded adjuvant formulations [17,29-31]. Previously, we also demonstrated that co-encapsulation of TLR9 and TLR3 ligands increased cytokine production (IL-6, type I and II IFNs) in vitro and induced longer IgG2c production after in vivo administration compared to the free and separately loaded dual-adjuvant formulations [17]. In another study in vivo administration of co-encapsulation of TLR4 and TLR7/8 ligands with an antigen elevated IgG2a levels more than 6.5 fold compared to the separately loaded liposomal mixtures. These studies indicated that by delivering both ligands into the same cell, coencapsulation assures ligands are activating and enhancing crosstalk between activated pathways [31]. Such simultaneous activation of pathways is important to observe synergism and overcome potential antagonism between signaling axes. A recent publication by FitzgeraldBocarsly et al. showed that pre-activation of STING pathway inhibits the TLR9-mediated interferon production from pDCs [32]. In addition, encapsulation of antigen with adjuvant within a delivery vehicle overcomes random antigen and adjuvant uptake by APCs, while increasing the internalization of exogenous antigen [33,34]. Based on these observations, we developed a pH-sensitive liposomal co-delivery system of CDN and CPG ODN combination along with the model tumor antigen OVA to release them into their target receptors after destabilization of lipids at late endosome with acidic pH (Fig. S6). We have previously defined that loading K-type CpG ODN within sterically stabilized cationic liposomes improved its immunostimulatory activity $[18,19]$. Therefore, we modified conventional sterically stabilized cationic liposome by using high lipid ratio of DOPE, which is a pH-sensitive lipid with fusogenic property. When we stimulated splenocytes with our construct containing suboptimal doses of K-ODN and CDN, we achieved significant elevation in both type I and II IFN productions. In contrast, free single or dual agonists were ineffective, even when used at higher doses, suggesting that liposomal formulation effectively delivers immunostimulatory agents to relevant cell(s).

Increased interferon secretion would lead the maturation of APCs and secretion of high amounts of Th1-biased cytokines [35]. Various 


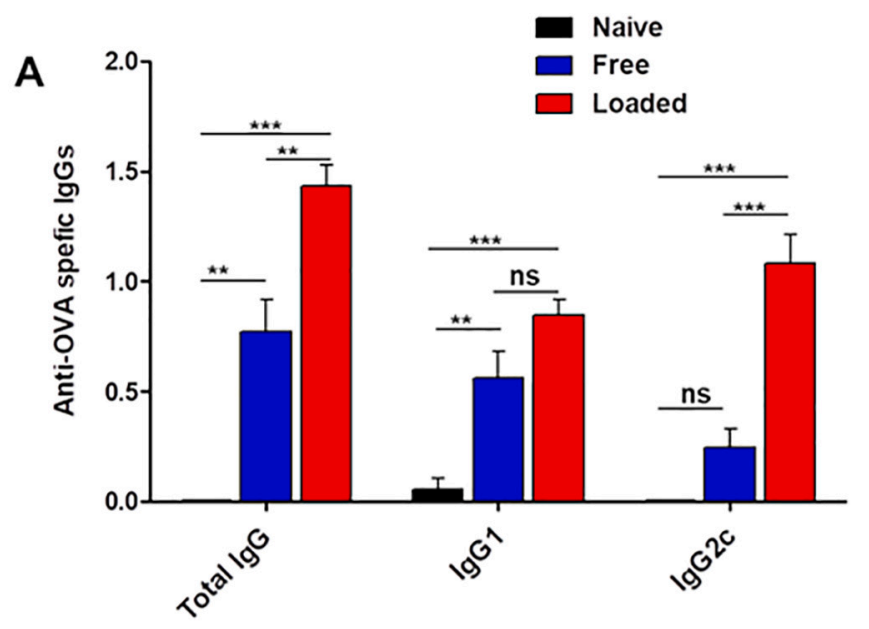

B



C

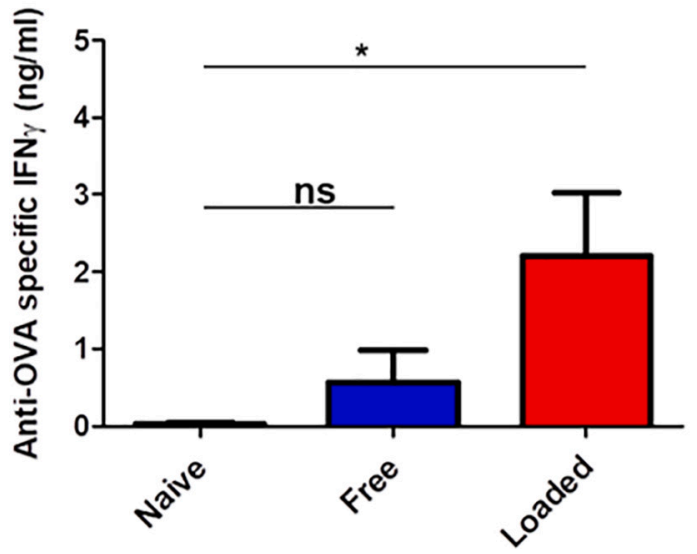

D

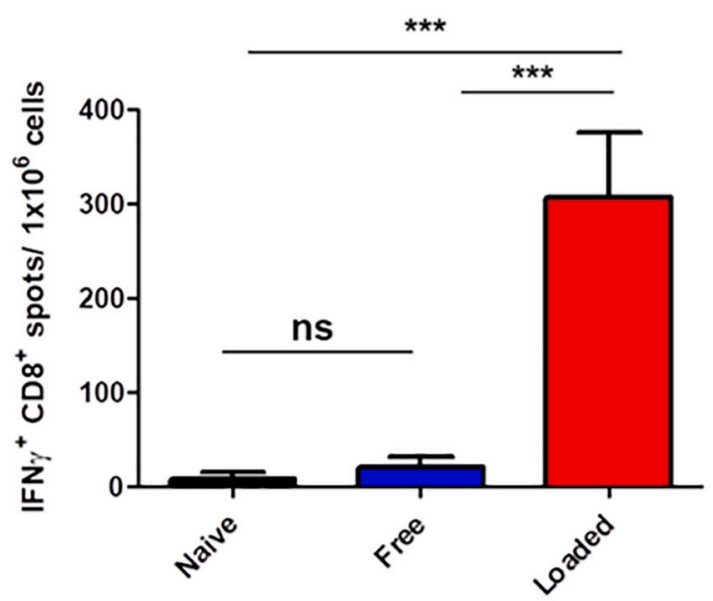

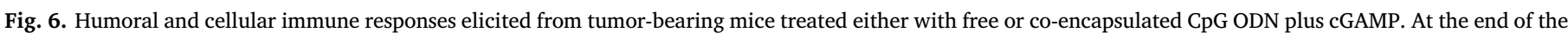

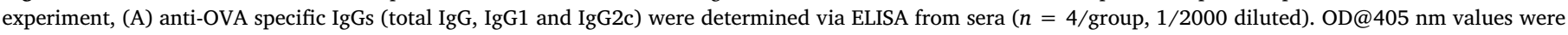

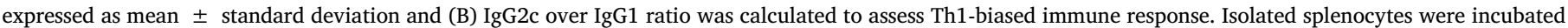

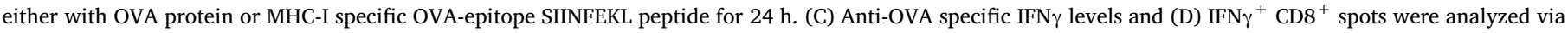

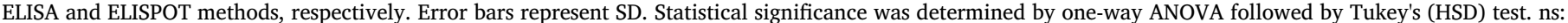
non-significant; * $p<0.05 ;{ }^{* *} p<0.01 ; * * * p<0.001$.

studies showed that encapsulated CDNs in delivery vehicles have capacity to elevate activity of APCs. In one study, the delivery of cGAMP incorporated within hydrogels magnified macrophage dependent IFN- $\beta$ secretion [36]. In another, particulate form of $c$-di-GMP activated APCs through elevation of co-stimulatory molecules [23]. Our study demonstrated that activating both STING and TLR9 receptors elevated IL12 and IL-6 productions from BM-DCs and BM-DMs, and promoted proliferation of macrophages in dual liposomal formulation. In addition, M1-like macrophages were generated as opposed to M2-type based on elevated IL-12/IL-10 ratio after stimulation of BM-DMs. Polarization of macrophages under the influence of the delivered ligands to M1-phenotype would be important during anti-cancer therapy to reprogram the suppressive tumor microenvironment.

Considering increased interferon secretion, APC activation and Th1 immune response, anti-tumor immune response would be improved with the administration of this dual adjuvant. Enhanced $\mathrm{CD} 8{ }^{+} \mathrm{T}$ cell
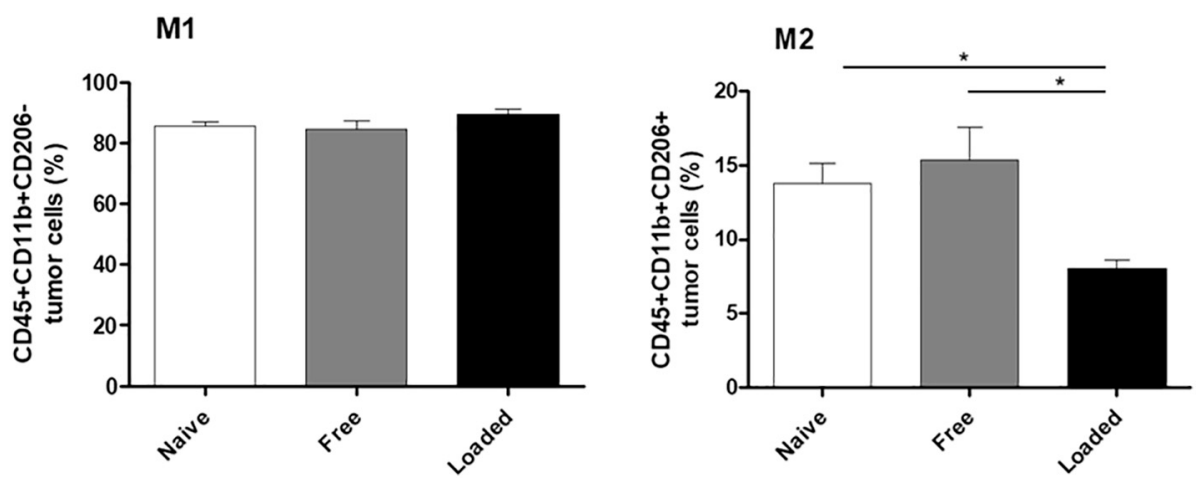

Fig. 7. Infiltrated macrophage sub-types in tumor micro niche of mice treated either with free or coencapsulated CpG ODN plus cGAMP. Isolated tumor cells were analyzed via flow cytometry after staining with fluorochrome-conjugated mAbs (CD45, CD11b, CD206). Error bars represent SEM. Statistical significance was determined by one-way ANOVA followed by Tukey's (HSD) test, * $p<0.05$. 
activity and anti-tumoral effect of CDNs have been studied previously. In a study by Corrales et al., high doses (25-50 $\mu$ g/injection) of synthetic CDNs regressed established tumors when administered intratumorally by tumor-resident DCs leading to IFN- $\beta$ production and subsequently priming T-cells [37]. In another study, adjuvant effect of cGAMP activated antigen-dependent humoral immune response with increased IgG1 production and induced antigen-specific IFN- $\gamma$ and IL-2 production from T cells [38]. Moreover, STING agonists induce migration of macrophages with high phagocytic activity and increased ISGs into tumor niche when administered intratumorally. Mainly, their anti-tumoral activity is dependent on macrophages and $\mathrm{CD}^{+} \mathrm{T}$ cell activation [39]. However, formulations in these studies used excessive amounts of cGAMP and administered intratumorally to elicit sufficient immune responses, which limits their translation to clinical settings.

Another synthetic STING agonist, 6-dimethylxanthenone-4-acetic acid (DMXAA or vadimezan), elicits re-education of M2-type macrophages into M1-type in metastatic tumors. However DMXAA is limited in its activity and fails to stimulate the human STING receptor as effectively as of 2'3'-cGAMP does [40,41]. Our liposomal formulation would boost the immune activation, which could overcome the suppressive environment existing in the tumor micro-niche and generate an inflammatory environment supporting tumor regression. In a melanoma tumor model, we demonstrated that the treatment of our liposomal formulation co-encapsulating CpG-ODN and cGAMP reduced tumor size by $\sim 70 \%$, boosted Th1-biased humoral response (IgG2c/ IgG1 > 1), increased IFN- $\gamma$ producing $\mathrm{CD}^{+} \mathrm{T}$ cells and reduced M2type macrophages at the tumor bed. We observed a strong antigenspecific $\mathrm{CD}^{+}{ }^{+} \mathrm{T}$ cell generation and reduced M2-macrophage frequency in the tumor bed following delivery of our liposomal dual agonists. Therefore, our data suggests that combination of the non-canonical 2'3' cGAMP together with CpG-ODN within liposomes would be a more potent immunotherapeutic agent, by replacing DMXAA. In summary, our findings identified an effective and safe method of delivering multiple immunotherapeutic agents to tumor-bearing mice and triggering reversal of tumor-specific immunosuppression. These observations indicate that combined activation of type I and type II IFN pathways can have a synergistic and beneficial effect and further provide the rationale of testing of combinational therapies for improved antitumor therapy.

\section{Conclusion}

We aimed to combine the properties of TLR9 and STING ligands by loading into $\mathrm{pH}$-sensitive liposomes which would eliminate the disadvantages of these agonists and design an adjuvant which could be translated successfully into the clinic. We tested in vitro immunostimulatory properties on splenocytes and bone marrow derived immune cells. Co-encapsulated CpG-ODN and CDNs within pH-sensitive modified SSCL synergistically increased type I and type II IFNs from splenocytes; while IL-12 and IL-6 production was substantially elevated from BM-DM and BM-DCs compared to unencapsulated free combinations. Dual delivery of ligands improved anti-cancer properties and subsided tumor growth approx. $70 \%$ by inducing a Th1-biased adaptive immune response and diminishing M2-macrophage population frequency in tumor bed. In conclusion, $\mathrm{pH}$ sensitive liposomes co-encapsulating both CpG-ODN and cGAMP is a promising immunotherapeutic agent that re-educate tumor environment, activate antigen-specific $\mathrm{CD}^{+}{ }^{+} \mathrm{T}$ cells and consequently regress tumor development via enhanced interferon and Th1-type immune response.

\section{Funding}

This work was partially supported by Scientific and Technological Research Council of Turkey (TUBITAK, Grant \#: SBAG-113S207 to I.G.), and Ministry of Development (Grant name: UMRAM-ASI, Project \#: 2015BSV302 to I.G.).

\section{Declaration of Competing Interest}

The authors confirm that there are no known conflicts of interest associated with this publication.

\section{Acknowledgements}

We thank Dr. Gamze Aykut for breeding and daily caring of our animals. We extend our appreciation to Dr. Dionysios C. Watson for their insightful comments and critical reading of our manuscript.

\section{Appendix A. Supplementary data}

Supplementary data to this article can be found online at https:// doi.org/10.1016/j.jconrel.2020.09.040.

\section{References}

[1] R.S. Riley, C.H. June, R. Langer, M.J. Mitchell, Delivery technologies for cancer immunotherapy, Nat. Rev. Drug Discov. 18 (2019) 175-196, https://doi.org/10. 1038/s41573-018-0006-Z.

[2] C.J. Desmet, K.J. Ishii, Nucleic acid sensing at the interface between innate and adaptive immunity in vaccination, Nat. Rev. Immunol. 12 (2012) 479-491, https:// doi.org/10.1038/nri3247.

[3] S. Pandey, T. Kawai, S. Akira, Microbial sensing by toll-like receptors and intracellular nucleic acid sensors, Cold Spring Harb. Perspect. Biol. 7 (2015) a016246, https://doi.org/10.1101/cshperspect.a016246.

[4] J. Scheiermann, D.M. Klinman, Clinical evaluation of $\mathrm{CpG}$ oligonucleotides as adjuvants for vaccines targeting infectious diseases and cancer, Vaccine. 32 (2014) 6377-6389, https://doi.org/10.1016/j.vaccine.2014.06.065.

[5] A.M. Krieg, CpG still rocks! Update on an accidental drug, Nucleic Acid Ther. 22 (2012) 77-89, https://doi.org/10.1089/nat.2012.0340.

[6] A.M. Krieg, Therapeutic potential of toll-like receptor 9 activation, Nat. Rev. Drug Discov. 5 (2006) 471-484, https://doi.org/10.1038/nrd2059.

[7] T. Junt, W. Barchet, Translating nucleic acid-sensing pathways into therapies, Nat. Rev. Immunol. 15 (2015) 529-544, https://doi.org/10.1038/nri3875.

[8] D. Verthelyi, K.J. Ishii, M. Gursel, F. Takeshita, D.M. Klinman, Human peripheral blood cells differentially recognize and respond to two distinct CpG motifs, J. Immunol. 166 (2001) 2372-2377, https://doi.org/10.4049/jimmunol.166.4.2372.

[9] K. Kobiyama, T. Aoshi, H. Narita, E. Kuroda, M. Hayashi, K. Tetsutani, S. Koyama, S. Mochizuki, K. Sakurai, Y. Katakai, Y. Yasutomi, S. Saijo, Y. Iwakura, S. Akira, C. Coban, K.J. Ishii, Nonagonistic Dectin-1 ligand transforms CpG into a multitask nanoparticulate TLR9 agonist, Proc. Natl. Acad. Sci. 111 (2014) 3086-3091, https://doi.org/10.1073/pnas.1319268111.

[10] B. Gungor, F.C. Yagci, G. Tincer, B. Bayyurt, E. Alpdundar, S. Yildiz, M. Ozcan, I. Gursel, M. Gursel, CpG ODN nanorings induce IFN from plasmacytoid dendritic cells and demonstrate potent vaccine adjuvant activity, Sci. Transl. Med. 6 (2014), https://doi.org/10.1126/scitranslmed.3007909 (235ra61-235ra61).

[11] A. de Titta, M. Ballester, Z. Julier, C. Nembrini, L. Jeanbart, A.J. van der Vlies, M.A. Swartz, J.A. Hubbell, Nanoparticle conjugation of CpG enhances adjuvancy for cellular immunity and memory recall at low dose, Proc. Natl. Acad. Sci. 110 (2013) 19902-19907, https://doi.org/10.1073/pnas.1313152110.

[12] S. Yildiz, E. Alpdundar, B. Gungor, T. Kahraman, B. Bayyurt, I. Gursel, M. Gursel, Enhanced immunostimulatory activity of cyclic dinucleotides on mouse cells when complexed with a cell-penetrating peptide or combined with CpG, Eur. J. Immunol. 45 (2015) 1170-1179, https://doi.org/10.1002/eji.201445133.

[13] H. Ishikawa, Z. Ma, G.N. Barber, STING regulates intracellular DNA-mediated, type I interferon-dependent innate immunity, Nature. 461 (2009) 788-792, https://doi. org/10.1038/nature08476.

[14] C. Shu, X. Li, P. Li, The mechanism of double-stranded DNA sensing through the cGAS-STING pathway, Cytokine Growth Factor Rev. 25 (2014) 641-648, https:// doi.org/10.1016/j.cytogfr.2014.06.006.

[15] T. Abe, G.N. Barber, Cytosolic-DNA-mediated, STING-dependent Proinflammatory gene induction necessitates canonical NF- B activation through TBK1, J. Virol. 88 (2014) 5328-5341 (doi:10.1128/JVI.00037-14).

[16] B. Temizoz, E. Kuroda, K. Ohata, N. Jounai, K. Ozasa, K. Kobiyama, T. Aoshi, K.J. Ishii, TLR9 and STING agonists synergistically induce innate and adaptive typeII IFN, Eur. J. Immunol. 45 (2015) 1159-1169, https://doi.org/10.1002/eji. 201445132.

[17] B. Bayyurt, G. Tincer, K. Almacioglu, E. Alpdundar, M. Gursel, I. Gursel, Encapsulation of two different TLR ligands into liposomes confer protective immunity and prevent tumor development, J. Control. Release 247 (2017) 134-144, https://doi.org/10.1016/j.jconrel.2017.01.004.

[18] E. Erikçi, M. Gursel, İ. Gürsel, Differential immune activation following encapsulation of immunostimulatory CpG oligodeoxynucleotide in nanoliposomes, Biomaterials. 32 (2011) 1715-1723, https://doi.org/10.1016/j.biomaterials.2010. 10.054.

[19] I. Gursel, M. Gursel, K.J. Ishii, D.M. Klinman, Sterically stabilized cationic liposomes improve the uptake and Immunostimulatory activity of $\mathrm{CpG}$ oligonucleotides, J. Immunol. 167 (2001) 3324-3328, https://doi.org/10.4049/jimmunol.167. 
6.3324 .

[20] R.M. Straubinger, N. Düzgünes, D. Papahadjopoulos, pH-sensitive liposomes mediate cytoplasmic delivery of encapsulated macromolecules, FEBS Lett. 179 (1985) 148-154, https://doi.org/10.1016/0014-5793(85)80210-6.

[21] X. Cao, Self-regulation and cross-regulation of pattern-recognition receptor signalling in health and disease, Nat. Rev. Immunol. 16 (2016) 35-50, https://doi.org/10. 1038/nri.2015.8.

[22] D.M. Underhill, Collaboration between the innate immune receptors dectin-1, TLRs, and nods, Immunol. Rev. 219 (2007) 75-87, https://doi.org/10.1111/j.1600-065X. 2007.00548.x.

[23] M.C. Hanson, M.P. Crespo, W. Abraham, K.D. Moynihan, G.L. Szeto, S.H. Chen, M.B. Melo, S. Mueller, D.J. Irvine, Nanoparticulate STING agonists are potent lymph node-targeted vaccine adjuvants, J. Clin. Invest. 125 (2015) 2532-2546, https://doi.org/10.1172/JCI79915.

[24] R.D. Junkins, M.D. Gallovic, B.M. Johnson, M.A. Collier, R. Watkins-Schulz, N. Cheng, C.N. David, C.E. McGee, G.D. Sempowski, I. Shterev, K. McKinnon, E.M. Bachelder, K.M. Ainslie, J.P.Y. Ting, A robust microparticle platform for a STING-targeted adjuvant that enhances both humoral and cellular immunity during vaccination, J. Control. Release 270 (2018) 1-13, https://doi.org/10.1016/j. jconrel.2017.11.030.

[25] D. Shae, K.W. Becker, P. Christov, D.S. Yun, A.K.R. Lytton-Jean, S. Sevimli, M. Ascano, M. Kelley, D.B. Johnson, J.M. Balko, J.T. Wilson, Endosomolytic polymersomes increase the activity of cyclic dinucleotide STING agonists to enhance cancer immunotherapy, Nat. Nanotechnol. 14 (2019) 269-278, https://doi.org/10. 1038/s41565-018-0342-5.

[26] T. Nakamura, H. Miyabe, M. Hyodo, Y. Sato, Y. Hayakawa, H. Harashima, Liposomes loaded with a STING pathway ligand, cyclic di-GMP, enhance cancer immunotherapy against metastatic melanoma, J. Control. Release 216 (2015) 149-157, https://doi.org/10.1016/j.jconrel.2015.08.026.

[27] H. Miyabe, M. Hyodo, T. Nakamura, Y. Sato, Y. Hayakawa, H. Harashima, A new adjuvant delivery system 'cyclic di-GMP/YSK05 liposome' for cancer immunotherapy, J. Control. Release 184 (2014) 20-27, https://doi.org/10.1016/j. jconrel.2014.04.004.

[28] S.R. Paliwal, R. Paliwal, S.P. Vyas, A review of mechanistic insight and application of pH-sensitive liposomes in drug delivery, Drug Deliv. 22 (2015) 231-242, https:// doi.org/10.3109/10717544.2014.882469.

[29] R. Madan-Lala, P. Pradhan, K. Roy, Combinatorial delivery of dual and triple TLR agonists via polymeric pathogen-like particles synergistically enhances innate and adaptive immune responses, Sci. Rep. 7 (2017) 2530, https://doi.org/10.1038/ s41598-017-02804-y.

[30] S.P. Kasturi, I. Skountzou, R.A. Albrecht, D. Koutsonanos, T. Hua, H.I. Nakaya, R. Ravindran, S. Stewart, M. Alam, M. Kwissa, F. Villinger, N. Murthy, J. Steel, J. Jacob, R.J. Hogan, A. García-Sastre, R. Compans, B. Pulendran, Programming the magnitude and persistence of antibody responses with innate immunity, Nature. 470 (2011) 543-547, https://doi.org/10.1038/nature09737.
[31] K.K. Short, S.M. Miller, L. Walsh, V. Cybulski, H. Bazin, J.T. Evans, D. Burkhart, Coencapsulation of synthetic lipidated TLR4 and TLR7/8 agonists in the liposomal bilayer results in a rapid, synergistic enhancement of vaccine-mediated humoral immunity, J. Control. Release 315 (2019) 186-196, https://doi.org/10.1016/j. jconrel.2019.10.025.

[32] P. Deb, J. Dai, S. Singh, E. Kalyoussef, P. Fitzgerald-Bocarsly, Triggering of the cGAS-STING pathway in human Plasmacytoid dendritic cells inhibits TLR9-mediated IFN production, J. Immunol. 205 (2020) 223-236, https://doi.org/10.4049/ jimmunol.1800933.

[33] A. Milicic, R. Kaur, A. Reyes-Sandoval, C.-K. Tang, J. Honeycutt, Y. Perrie, A.V.S. Hill, Small cationic DDA:TDB liposomes as protein vaccine adjuvants obviate the need for TLR agonists in inducing cellular and humoral responses, PLoS One 7 (2012) e34255, https://doi.org/10.1371/journal.pone.0034255.

[34] E. Schlosser, M. Mueller, S. Fischer, S. Basta, D.H. Busch, B. Gander, M. Groettrup, TLR ligands and antigen need to be coencapsulated into the same biodegradable microsphere for the generation of potent cytotoxic T lymphocyte responses, Vaccine. 26 (2008) 1626-1637, https://doi.org/10.1016/j.vaccine.2008.01.030.

[35] B.S. Parker, J. Rautela, P.J. Hertzog, Antitumour actions of interferons: implications for cancer therapy, Nat. Rev. Cancer 16 (2016) 131-144, https://doi.org/10.1038/ nrc.2016.14.

[36] E. Lee, H.-E. Jang, Y.Y. Kang, J. Kim, J.-H. Ahn, H. Mok, Submicron-sized hydrogels incorporating cyclic dinucleotides for selective delivery and elevated cytokine release in macrophages, Acta Biomater. 29 (2016) 271-281, https://doi.org/10. 1016/j.actbio.2015.10.025.

[37] T. Ohkuri, A. Kosaka, K. Ishibashi, T. Kumai, Y. Hirata, K. Ohara, T. Nagato, K. Oikawa, N. Aoki, Y. Harabuchi, E. Celis, H. Kobayashi, Intratumoral administration of cGAMP transiently accumulates potent macrophages for anti-tumor immunity at a mouse tumor site, Cancer Immunol. Immunother. 66 (2017) 705-716, https://doi.org/10.1007/s00262-017-1975-1.

[38] X.-D. Li, J. Wu, D. Gao, H. Wang, L. Sun, Z.J. Chen, Pivotal roles of cGAS-cGAMP signaling in antiviral defense and immune adjuvant effects, Science (80-.) 341 (2013) 1390-1394, https://doi.org/10.1126/science.1244040.

[39] C.M. Downey, M. Aghaei, R.A. Schwendener, F.R. Jirik, DMXAA causes tumor sitespecific vascular disruption in murine non-small cell lung cancer, and like the endogenous non-canonical cyclic dinucleotide STING agonist, 2'3'-cGAMP, induces M2 macrophage repolarization, PLoS One 9 (2014) e99988, https://doi.org/10. 1371/journal.pone.0099988.

[40] X. Zhang, H. Shi, J. Wu, X. Zhang, L. Sun, C. Chen, Z.J. Chen, Cyclic GMP-AMP containing mixed Phosphodiester linkages is an endogenous high-affinity ligand for STING, Mol. Cell 51 (2013) 226-235, https://doi.org/10.1016/j.molcel.2013.05. 022 .

[41] H. Shi, J. Wu, Z.J. Chen, C. Chen, Molecular basis for the specific recognition of the metazoan cyclic GMP-AMP by the innate immune adaptor protein STING, Proc. Natl. Acad. Sci. 112 (2015) 8947-8952, https://doi.org/10.1073/pnas. 1507317112 . 